\title{
Werteorientierung und ihre sechs unterschiedlichen Definitionen
}

Unser Begriff „Wert(e)orientierung“ bietet aufgrund der unterschiedlichen Zielgruppen auch mehrere recht unterschiedliche Bedeutungsmöglichkeiten.

Wir verstehen darunter:

- in der christlichen Tradition die christlich geprägten Vorgaben (z. B. die zehn Gebote),

- in der Politik eine gemeinsame Interessenvertretung (z. B. Interessenverbände, EU oder Nato),

- in der Wirtschaft den unternehmerischen Gesamtnutzen - Value Based Management,

- in unserer Gesellschaft die Einhaltung gesellschaftlicher und rechtlicher Regeln,

- in der Partnerschaft Liebe, Toleranz, Vertrauen und Verantwortung, ... die wir mit unserem Partner erleben wollen,

- in der Führungslehre eine Verknüpfung der menschlichen Werte mit den Management- und Führungsinstrumenten (zum Aufbau von langfristig tragfähigen und belastbaren Arbeitsbeziehungen).

Die ersten dieser vier Definitionen sind Wertvorgaben und Anweisungen, die eingelöst werden sollen. Das, was wir mit Wertewandel beschreiben, wird deutlicher, wenn wir uns die letzten beiden Definitionen anschauen. Hier erleben wir eine Loslösung von der klassischen Werteorientierung im Hinblick auf die Aufgabenbewältigung. Sie reicht von der Zielvorgabe über die Zielvereinbarung hin zu einer Einbeziehung der menschlichen Werte der Mitarbeiter, Partner und Kunden in unserem Zusammenwirken. Der Wertewandel stellt sich als Folge einer Renaissance oder „Wiedergeburt“ der menschlichen Werte in unserem Zusammenwirken dar. 